\title{
Article
}

\section{Spectral conditions for the Bipancyclic Bipartite graphs}

\section{Rao Li}

Department of mathematical sciences, University of South Carolina Aiken, Aiken, SC 29801, USA.; raol@usca.edu

Academic Editor: Muhammad Imran

Received: 12 July 2021; Accepted: 30 July 2021; Published: 31 August 2021

Abstract: Let $G=(X, Y ; E)$ be a bipartite graph with two vertex partition subsets $X$ and $Y$. G is said to be balanced if $|X|=|Y|$ and $G$ is said to be bipancyclic if it contains cycles of every even length from 4 to $|V(G)|$. In this note, we present spectral conditions for the bipancyclic bipartite graphs.

Keywords: Spectral condition; Bipancyclic Bipartite Graphs.

MSC: 05C50, 05C45.

\section{Introduction}

W e consider only finite undirected graphs without loops or multiple edges. Notation and terminology not defined here follow that in [1]. Let $G=(V(G), E(G))$ be a graph. The graph $G$ is said to be Hamiltonian if it contains a cycle of length $|V(G)|$. The graph $G$ is said to be pancyclic if it contains cycles of every length from 3 to $|V(G)|$. Let $G=\left(V_{1}(G), V_{2}(G) ; E(G)\right)$ be a bipartite graph with two vertex partition subsets $V_{1}(G)$ and $V_{2}(G)$. The bipartite graph $G$ is said to be semiregular bipartite if all the vertices in $V_{1}(G)$ have the same degree and all the vertices in $V_{2}(G)$ have the same degree. The bipartite graph $G$ is said to be balanced if $\left|V_{1}\right|=\left|V_{2}\right|$. Clearly, if a bipartite graph is Hamiltonian, then it must be balanced. The bipartite graph $G$ is said to be bipancyclic if it contains cycles of every even length from 4 to $|V(G)|$. The balanced bipartite graph $G_{1}=(A, B ; E)$ of order $2 n$ with $n \geq 4$ is defined as follows: $A=\left\{a_{1}, a_{2}, \ldots, a_{n}\right\}, B=\left\{b_{1}, b_{2}, \ldots, b_{n}\right\}$, and $E=\left\{a_{i} b_{j}: 1 \leq i \leq 2,(n-1) \leq j \leq n\right\} \cup\left\{a_{i} b_{j}: 3 \leq i \leq n, 1 \leq j \leq n\right\}$. Notice that $G_{1}$ is not Hamiltonian.

The eigenvalues of a graph $G$, denoted $\lambda_{1}(G) \geq \lambda_{2}(G) \geq \cdots \geq \lambda_{n}(G)$, are defined as the eigenvalues of its adjacency matrix $A(G)$. Let $D(G)$ be a diagonal matrix such that its diagonal entries are the degrees of vertices in a graph $G$. The Laplacian matrix of a graph $G$, denoted $L(G)$, is defined as $D(G)-A(G)$, where $A(G)$ is the adjacency matrix of $G$. The eigenvalues $\mu_{1}(G) \geq \mu_{2}(G) \geq \cdots \geq \mu_{n-1}(G) \geq \mu_{n}(G)=0$ of $L(G)$ are called the Laplacian eigenvalues of $G$. The second smallest Laplacian eigenvalue $\mu_{n-1}(G)$ is also called the algebraic connectivity of the graph $G$ (see [2]). The signless Laplacian matrix of a graph $G$, denoted $Q(G)$, is defined as $D(G)+A(G)$, where $A(G)$ is the adjacency matrix of $G$. The eigenvalues $q_{1}(G) \geq q_{2}(G) \geq \cdots \geq q_{n}(G)$ of $Q(G)$ are called the signless Laplacian eigenvalues of $G$.

Yu et al., in [3] obtained some spectral conditions for the pancyclic graphs. Motivated by the results in [3], we present spectral conditions for the bipancyclic bipartite graphs. The main results are as follows:

Theorem 1. Let $G=(X, Y ; E)$ be a connected balanced bipartite graph of order $2 n$ with $n \geq 4$, e edges, and $\delta \geq 2$. If $\lambda_{1} \geq \sqrt{n^{2}-2 n+4}$, then $G$ is bipancyclic.

Theorem 2. Let $G=(X, Y ; E)$ be a connected balanced bipartite graph of order $2 n$ with $n \geq 4$, e edges, and $\delta \geq 2$. If

$$
\mu_{n-1} \geq \frac{2\left(n^{2}-2 n+4\right)}{n},
$$

then $\mathrm{G}$ is bipancyclic. 
Theorem 3. Let $G=(X, Y ; E)$ be a connected balanced bipartite graph of order $2 n$ with $n \geq 4$, e edges, and $\delta \geq 2$. If

$$
q_{1} \geq \frac{2\left(n^{2}-n+2\right)}{n}
$$

then $G$ is bipancyclic.

\section{Lemmas}

In order to prove the theorems above, we need the following results as our lemmas:

Lemma 1 is the main result in [4].

Lemma 1. Let $G=(X, Y ; E)$ be a balanced bipartite graph of order $2 n$ with $n \geq 4$. Suppose that $X=\left\{x_{1}, x_{2}, \ldots, x_{n}\right\}$, $Y=\left\{y_{1}, y_{2}, \ldots, y_{n}\right\}, d\left(x_{1}\right) \leq d\left(x_{2}\right) \leq \cdots \leq d\left(x_{n}\right)$, and $d\left(y_{1}\right) \leq d\left(y_{2}\right) \leq \cdots \leq d\left(y_{n}\right)$. If

$$
d\left(x_{k}\right) \leq k<n \Longrightarrow d\left(y_{n-k}\right) \geq n-k+1,
$$

then $G$ is bipancyclic.

Lemma 2 below follows from Proposition 2.1 in [5]:

Lemma 2. Let $G$ be a connected bipartite graph of order $n \geq 2$ and $e \geq 1$ edges. Then $\lambda_{1} \leq \sqrt{e}$. If $\lambda_{1}=\sqrt{e}$, then $G$ is a complete bipartite graph $K_{s, t}$, where e $=s t$.

Lemma 3 below is Lemma 4.1 in [2]:

Lemma 3. Let $G$ be a noncomplete graph. Then $\mu_{n-1} \leq \kappa$, where $\kappa$ is the vertex connectivity of $G$.

Lemma 4 below is Theorem 2.9 in [6]:

Lemma 4. Let $G$ be a balanced bipartite graph of order $2 n$ and e edges. Then $q(G) \leq \frac{e}{n}+n$.

Lemma 5 below is Lemma 2.3 in [7]:

Lemma 5. Let G be a connected graph. Then

$$
q_{1} \leq \max \left\{d(u)+\frac{\sum_{v \in N(u)} d(v)}{d(u)}: u \in V\right\},
$$

with equality holding if and only if $G$ is either semiregular bipartite or regular.

Lemma 6. Let $G=(X, Y ; E)$ be a balanced bipartite graph of order $2 n$ with $n \geq 4$, e edges, and $\delta \geq 2$. If e $\geq n^{2}-2 n+4$, then $G$ is bipancyclic or $G=G_{1}$.

Proof. Without loss of generality, we assume that $X=\left\{x_{1}, x_{2}, \ldots, x_{n}\right\}, Y=\left\{y_{1}, y_{2}, \ldots, y_{n}\right\}, d\left(x_{1}\right) \leq d\left(x_{2}\right) \leq \ldots \leq$ $d\left(x_{n}\right)$, and $d\left(y_{1}\right) \leq d\left(y_{2}\right) \leq \cdots \leq d\left(y_{n}\right)$. Suppose $G$ is not bipancyclic. Then Lemma 1 implies that there exists an integer $k$ such that $1 \leq k<n, d\left(x_{k}\right) \leq k$, and $d\left(y_{n-k}\right) \leq n-k$. Thus

$$
\begin{aligned}
2 n^{2}-4 n+8 & \leq 2 e \\
& =\sum_{i=1}^{n} d\left(x_{i}\right)+\sum_{i=1}^{n} d\left(y_{i}\right) \\
& \leq k^{2}+(n-k) n+(n-k)^{2}+k n \\
& =2 n^{2}-4 n+8-(k-2)(2 n-2 k-4)
\end{aligned}
$$

Since $\delta \geq 2$, we have that $k \neq 1$. Therefore we have the following possible cases.

Case 1. $k=2$. 
In this case, all the inequalities in the above arguments now become equalities. Thus $d\left(x_{1}\right)=d\left(x_{2}\right)=2$, $d\left(x_{3}\right)=\cdots=d\left(x_{n}\right)=n, d\left(y_{1}\right)=\cdots=d\left(y_{n-2}\right)=n-2$, and $d\left(y_{n-1}\right)=d\left(y_{n-2}\right)=n$. Hence $G=G_{1}$.

Case 2. $(2 n-2 k-4)=0$.

In this case, we have $n=k+2$ and all the inequalities in the above arguments now become equalities. Thus $d\left(x_{1}\right)=\cdots=d\left(x_{n-2}\right)=n-2, d\left(x_{n-1}\right)=d\left(x_{n}\right)=n, d\left(y_{1}\right)=d\left(y_{2}\right)=n-2$, and $d\left(y_{3}\right)=\cdots=d\left(y_{n-2}\right)=n$. Hence $G=G_{1}$.

Case 3. $k \geq 3$ and $2 n-2 k-4<0$.

In this case, we have that $n<k+2$, namely, $n \leq k+1$. Since $k<n$, we have $k=n-1$. This implies that $d\left(y_{1}\right) \leq 1$, contradicting to the assumption of $\delta \geq 2$.

This completes the proof of Lemma 6.

\section{Proofs}

Proof of Theorem 1. Let $G$ be a graph satisfying the conditions in Theorem 1. Then we, from Lemma 2, we have

$$
\sqrt{n^{2}-2 n+4} \leq \lambda_{1} \leq \sqrt{e} .
$$

Thus $e \geq n^{2}-2 n+4$. Therefore by Lemma 6 we have that $G$ is bipancyclic or $G=G_{1}$.

If $G=G_{1}$, then $e=n^{2}-2 n+4$. Hence

$$
\sqrt{n^{2}-2 n+4} \leq \lambda_{1} \leq \sqrt{e}=\sqrt{n^{2}-2 n+4} .
$$

So $\lambda_{1}=\sqrt{e}$. Lemma 2 implies that $G_{1}$ is a complete bipartite graph, a contradiction.

This completes the proof of Theorem 1.

Proof of Theorem 2. Let $G$ be a graph satisfying the conditions in Theorem 2. Then we, from Lemma 3, we have

$$
\frac{2\left(n^{2}-2 n+4\right)}{n} \leq \mu_{n-1} \leq \kappa \leq \delta \leq \frac{2 e}{n}
$$

Thus $e \geq n^{2}-2 n+4$. Therefore by Lemma 6 we have that $G$ is bipancyclic or $G=G_{1}$.

If $G=G_{1}$, then $e=n^{2}-2 n+4$. Hence

$$
\frac{2\left(n^{2}-2 n+4\right)}{n} \leq \mu_{n-1} \leq \kappa \leq \delta \leq \frac{2 e}{n}=\frac{2\left(n^{2}-2 n+4\right)}{n} .
$$

This implies that $G_{1}$ is a regular graph, a contradiction.

This completes the proof of Theorem 2.

Proof of Theorem 3. Let $G$ be a graph satisfying the conditions in Theorem 3. Then we, from Lemma 4, we have

$$
\frac{2\left(n^{2}-n+2\right)}{n} \leq q_{1} \leq \frac{e}{n}+n .
$$

Thus $e \geq n^{2}-2 n+4$. Therefore by Lemma 6 we have that $G$ is bipancyclic or $G=G_{1}$.

If $G=G_{1}$, then $e=n^{2}-2 n+4$. Therefore

$$
\frac{2\left(n^{2}-n+2\right)}{n} \leq q_{1} \leq \frac{e}{n}+n=\frac{n^{2}-2 n+4}{n}+n=\frac{2\left(n^{2}-n+2\right)}{n} .
$$

Hence

$$
q_{1}=\frac{2\left(n^{2}-n+2\right)}{n}
$$

It can be verified that

$$
\max \left\{d(u)+\frac{\sum_{v \in N(u)} d(v)}{d(u)}: u \in V\right\}=\frac{2\left(n^{2}-n+2\right)}{n}=q_{1} .
$$

Thus Lemma 5 implies that $G_{1}$ is semiregular or regular, a contradiction.

This completes the proof of Theorem 3 . 
Conflicts of Interest: "The author declares no conflict of interest."

\section{References}

[1] Bondy, J. A., \& Murty, U. S. R. (1976). Graph Theory with Applications. Macmillan, London and Elsevier, New York.

[2] Fiedler, M. (1973). Algebraic connectivity of graphs, Czechoslovak Mathematical Journal, 23, 298-305.

[3] Yu, G., Yu, T., Shu, A., \& Xia, X. (2020). Some sufficient conditions on pancyclic graphs. https://arxiv.org/pdf/1809.09897.pdf, retrieved on July 28, 2020.

[4] Mitchem, J., \& Schmeichel, E. F. (1982). Bipartite graphs with all even lengths. Journal of Graph Theory, 6, $429-439$.

[5] Bhattacharya, A., Friedland, S., \& Peled, U. (2008). On the first eigenvalue of bipartite graphs. The Electronic Journal of Combinatorics, 15, \#R 144.

[6] Li, B., \& Ning, B. (2020). Spectral analogues of Erdős' and Moon-Moser's theorems on Hamilton cycles. https://arxiv.org/pdf/1504.03556.pdf, retrieved on July 28, 2020.

[7] Feng, L., \& Yu, G. (2009). On three conjectures involving the signless Laplacian spectral radius of graphs. Publications de l'Institut Mathématique, 85, 35-38.

[8] Cvetković, D., Doob, M., \& Sachs, H. (1995). Spectra of Graphs, 3rd Edition. Johann Ambrosius Barth. 